\title{
Improving proton therapy by metal-containing nanoparticles: nanoscale insights
}

\author{
This article was published in the following Dove Press journal: \\ International Journal of Nanomedicine \\ 15 April 2016 \\ Number of times this article has been viewed
}

\author{
Thomas Schlathölter' \\ Pierre Eustache ${ }^{2}$ \\ Erika Porcel ${ }^{2}$ \\ Daniela Salado 2 \\ Lenka Stefancikova ${ }^{2}$ \\ Olivier Tillement ${ }^{3}$ \\ Francois Lux ${ }^{3}$ \\ Pierre Mowat ${ }^{3}$ \\ Aleksandra K Biegun ${ }^{4}$ \\ Marc-Jan van Goethem ${ }^{4}$ \\ Hynd Remita ${ }^{5}$ \\ Sandrine Lacombe ${ }^{2}$ \\ 'Zernike Institute for Advanced \\ Materials, University of Groningen, \\ Groningen, the Netherlands; ${ }^{2}$ Institut \\ des Sciences Moléculaires d'Orsay \\ (ISMO), Univ. Paris Sud, CNRS, \\ Université Paris Saclay, Orsay Cedex, \\ ${ }^{3}$ Institut Lumière Matière, Villeurbanne \\ Cedex, France; ${ }^{4}$ Kernfysisch Versneller \\ Instituut - Center for Advanced \\ Radiation Technology (KVI-CART), \\ University of Groningen, Groningen, \\ the Netherlands; ${ }^{5}$ Laboratoire de \\ Chimie Physique, Universite Paris-Sud, \\ Orsay Cedex, France
}

Correspondence: Thomas Schlathölter Zernike Institute for Advanced Materials, University of Groningen, Nijenborgh 4, 9747AG Groningen, the Netherlands

$\mathrm{Tel}+3 \mid 503635099$

Fax +3I 503634400

Email t.a.schlatholter@rug.nl

\begin{abstract}
The use of nanoparticles to enhance the effect of radiation-based cancer treatments is a growing field of study and recently, even nanoparticle-induced improvement of proton therapy performance has been investigated. Aiming at a clinical implementation of this approach, it is essential to characterize the mechanisms underlying the synergistic effects of nanoparticles combined with proton irradiation. In this study, we investigated the effect of platinum- and gadolinium-based nanoparticles on the nanoscale damage induced by a proton beam of therapeutically relevant energy $(150 \mathrm{MeV})$ using plasmid DNA molecular probe. Two conditions of irradiation $(0.44$ and $3.6 \mathrm{keV} / \mu \mathrm{m})$ were considered to mimic the beam properties at the entrance and at the end of the proton track. We demonstrate that the two metal-containing nanoparticles amplify, in particular, the induction of nanosize damages $(>2 \mathrm{~nm}$ ) which are most lethal for cells. More importantly, this effect is even more pronounced at the end of the proton track. This work gives a new insight into the underlying mechanisms on the nanoscale and indicates that the addition of metal-based nanoparticles is a promising strategy not only to increase the cell killing action of fast protons, but also to improve tumor targeting.
\end{abstract}

Keywords: gadolinium-based nanoparticles, platinum nanoparticles, nanosensitization, theranostics

\section{Introduction}

In the last decade, important developments in nanomedicine have opened up new perspectives for tumor diagnostics and targeted cancer therapy. ${ }^{1}$ Different studies have demonstrated that nanoparticles (NPs) conjugated with specific ligands can efficiently target malignant tumor cells. ${ }^{2-4}$ In addition, NPs composed of high-Z elements such as noble metals or lanthanides amplify the effect of radiation treatment. ${ }^{5}$ This phenomenon, which was first evidenced for platinum complexes combined with highenergy photons ${ }^{6,7}$ and fast ions,${ }^{8}$ was later demonstrated for gold NPs (AuNPs) ${ }^{9}$ and platinum NPs (PtNPs) ${ }^{10}$ in combination with photons or fast ions. Subsequent simulation studies have led to first insights into this amplification effect. ${ }^{11-14}$ Furthermore, nanostructures were found to not only amplify radiation effects, but also improve in situ tumor diagnostics. In particular, gadolinium-based NPs (GdBNs) are magnetic resonance imaging active agents for which amplification of cell killing was observed using high-energy photons ${ }^{15-17}$ and carbon ions as incident radiation. ${ }^{18}$ The new properties of these multimodal compounds open up promising perspectives to implement theranostics (therapy and diagnosis based on a single compound) in radiation-based cancer therapy.

In parallel, the use of fast ions (protons and carbon ions) has proven to be another promising approach to treat cancer. Fast ions are predominantly used for treatment of 
solid tumors embedded in sensitive tissues (eg, in the region of the head, neck, or other sensitive organs), for radioresistant tumors, and in case of pediatric patients. ${ }^{19}$ At present, numerous proton therapy centers are under construction throughout the world. Compared to photons, the main advantage of ions stems from their unique dose-depth distribution, which peaks in a well-defined depth at the end of the particle tracks (Bragg peak), enabling superior dose deposition in the tumor. The tissues located in front of and behind the tumor, therefore, receive low doses. However, for clinical use, the ion beam needs to be energy modulated to implement a spread-out Bragg peak that ensures constant physical dose over the total tumor volume. Unfortunately, this energy modulation increases the dose deposition in tissues in front of the tumor. The addition of NPs in the tumor has been proposed as a new strategy to enhance selective cell killing in the tumor and, thus, overcome the limitation of hadron therapy. Few studies have demonstrated the efficiency of high-Z NPs to amplify the effects of fast protons. In particular, Kim et $\mathrm{al}^{20}$ reported that cell survival decreases upon irradiation with $45 \mathrm{MeV}$ protons, when tumor cells are loaded with gold or iron NPs. Polf et $\mathrm{al}^{21}$ observed a similar decrease in prostate tumor cell survival when loaded with AuNPs and irradiated with $160 \mathrm{MeV}$ protons. Recent in vivo studies performed with $45 \mathrm{MeV}$ protons demonstrated that the addition of metallic NPs results in a faster tumor remission in mice. ${ }^{22}$

Even though the beneficial effect of NPs on cell killing induced by fast proton radiation is established, the underlying nanoscale mechanisms remain poorly understood. Some studies ascribed the enhancement of radiation effects by NPs as due to ion-induced release of electron bursts, subsequently leading to the production of reactive oxygen species (ROS) clusters in the medium. ${ }^{10}$ In contrast, Kim et $\mathrm{al}^{20}$ first attributed this effect to proton-induced X-ray emission. Despite the fact that this explanation was later dismissed, there is still an urgent need to better understand the nanoscale processes induced in the presence of NPs.

The present study aims at shedding light on the elementary mechanisms that govern the effects of nanoagents when combined with fast protons. It is commonly accepted that nanosize lesions, also called complex damages, are the most lethal for the cells. ${ }^{23}$ In this work, we quantified the efficacy of $3 \mathrm{~nm}$ platinum $(Z=78)$ and $5 \mathrm{~nm}$ gadolinium $(Z=64)$ based NPs to amplify nanosize lesions upon fast proton irradiation. The plasmid pBR322 was used as a molecular probe to quantify simple and complex damages in biomolecules. ${ }^{24}$ Note that here pBR322 is not considered as a model for nuclear DNA. The experiments were performed with ion beams, which mimic the beam at the entrance of the ion track (linear energy transfer $[\mathrm{LET}]=0.44 \mathrm{keV} / \mu \mathrm{m}$ ) and the beam at the end of the track $(\mathrm{LET}=3.6 \mathrm{keV} / \mu \mathrm{m})$. We also characterized the influence of hydroxyl radicals on the NP effects.

\section{Materials and methods Samples}

The 4,361 base pair plasmid pBR322 (Euromedex, Souffelweyersheim, France) (molecular weight $\left.=2.83 \times 10^{6} \mathrm{Da}\right)$ was diluted in a Tris-ethylenediaminetetraacetic acid (Tris-EDTA or TE) buffer solution (10 mM Tris- $\mathrm{HCl}[\mathrm{pH}=7.6]$ and 1 $\mathrm{mmol} / \mathrm{L}$ EDTA) that mimics the medium conditions of living cells. Prior to irradiation, $95 \%$ of the plasmids were found to be supercoiled and 5\% had circular conformation, indicative of a single-strand break (SSB). The linear conformation, which is indicative of double-strand breaks (DSBs), was absent. Here, DSB corresponds mainly to two face-to-face SSB lesions separated by $<10$ base pairs. Hence, the production of DSB is used to probe the induction of nanosize molecular damage (also called complex damage). After irradiation, the three plasmid conformations were separated and quantified by agarose gel electrophoresis, as described elsewhere. ${ }^{10}$

PtNPs were synthesized by radiolysis, a unique and clean method. Synthesis takes place in water without addition of reducing agents. In the process, solvated electrons and reducing radicals $\left(\mathrm{H}^{\circ}\right)$ reduce the metal precursors homogeneously, leading to metal NPs that are homogeneous in size. ${ }^{10}$ Briefly, tetraammine platinum (II) chloride salt $\left[\mathrm{Pt}\left(\mathrm{NH}_{3}\right)_{4}\right] \mathrm{Cl}_{2} \cdot \mathrm{H}_{2} \mathrm{O}$ $\left(10^{-4} \mathrm{~mol} / \mathrm{L}\right)$ (Sigma-Aldrich Co., St Louis, MO, USA) was used as the precursor and polyacrylic acid $\left(10^{-2} \mathrm{~mol} / \mathrm{L}\right)$ (SigmaAldrich Co.) as the stabilizer. After deaeration, the solutions were irradiated by gamma rays provided by a panoramic $\mathrm{Co}^{60}$ gamma source (LCP, Orsay, France). The platinum complex was reduced by solvated electrons and $\mathrm{H}^{\bullet}$ radicals stemming from radiolysis. Complete reduction of platinum (+II) in platinum (0) was obtained for a dose of 1,000 Gy. Homogeneous NPs of $3 \mathrm{~nm}$ diameter were produced. ${ }^{10}$ The PtNPs contain on an average 1,000 platinum atoms. Stored under dark conditions at $4^{\circ} \mathrm{C}$, PtNPs are stable for several weeks. For the present experiments, PtNPs were diluted in ultrapure water to obtain a final platinum concentration of $4.23 \times 10^{-5} \mathrm{~mol} / \mathrm{L}$.

GdBNs were synthesized as described elsewhere. ${ }^{25}$ The nanoparticles are composed of a polysiloxane core surrounded by gadolinium chelates. GdBNs are functionalized with 1,4,7,10-tetraazacyclododecane-1,4,7,10-tetraacetic acid. Each NP contains approximately ten gadolinium atoms and has a diameter of $\sim 3 \mathrm{~nm}$. Stored under dark conditions at $4^{\circ} \mathrm{C}$, GdBNs are stable for several months. 
For the present experiments, GdBNs were diluted with ultrapure water to obtain a final gadolinium concentration of $4.23 \times 10^{-5} \mathrm{~mol} / \mathrm{L}$.

Some experiments were performed in the presence of dimethyl sulfoxide (DMSO) $1 \mathrm{~mol} / \mathrm{L}$, an efficient hydroxyl radical scavenger. ${ }^{26}$

\section{Proton beam irradiation}

The $150 \mathrm{MeV}$ proton beam was provided by the AGOR cyclotron located at the Kernfysisch Versneller Instituut Center for Advanced Radiation Technology in Groningen (the Netherlands). The beam was scattered using a doublescatter foil setup to produce a homogeneous irradiation field with a variation of $<3 \%$ over $70 \mathrm{~mm}$ field diameter.

For irradiation, the samples were placed in a water container at a depth of $30 \mathrm{~mm}$ measured along the beam (Figure 1). This geometry was used to avoid material density changes in the sample region and prevent dosimetry artifacts. The location of the samples along the dose-depth curve was controlled with a precision of $\sim 1 \mathrm{~mm}$, so that the dose deviation was $<3 \%$ over a diameter of $3.5 \mathrm{~cm}$.

The averaged LET at each position was calculated using Geant 4 simulations (version 4.10.00.p01, with the QGSP_BERT model for hadronic reactions and the standard electromagnetic interaction model with option 3). ${ }^{27} \mathrm{We}$ found $0.44 \mathrm{keV} / \mu \mathrm{m}$ at the entrance channel and $3.6 \mathrm{keV} / \mu \mathrm{m}$ at the Bragg peak.
The dose delivered to the samples was determined by recording the signal from an ionization chamber that served as an online beam monitor during sample irradiation. For calibration of the ionization chamber to an absolute dose rate, a calibrated Markus chamber was used (PTW Freiburg $\mathrm{GmbH}$, Freiburg, Germany). During the calibration procedure, the samples were removed and the Markus chamber was positioned at the sample position. This procedure is proposed in the respective protocol of the International Atomic Energy Agency. ${ }^{28}$

Four sets of experiments were performed with samples containing 1) metal-free plasmid samples used as controls, 2) plasmids and PtNPs, 3) plasmids and GdBNs, and 4) plasmids and DMSO (with or without NPs). These experiments were performed under atmospheric conditions at room temperature with doses ranging from 0 to $350 \mathrm{~Gy}$ for most of the experiments and up to $800 \mathrm{~Gy}$ in the presence of DMSO. The dose rate was close to $30 \mathrm{~Gy} / \mathrm{min}$.

\section{Analysis}

The samples were analyzed by agarose gel electrophoresis as detailed elsewhere. ${ }^{10,29}$ After migration at room temperature, the gel was stained with ethidium bromide and exposed to ultraviolet light $(312 \mathrm{~nm})$. The plasmid fluorescence was recorded with a charged coupled device camera. The densitometry was performed using the Quantity One software package (Bio-Rad Laboratories Inc., Hercules, CA, USA).

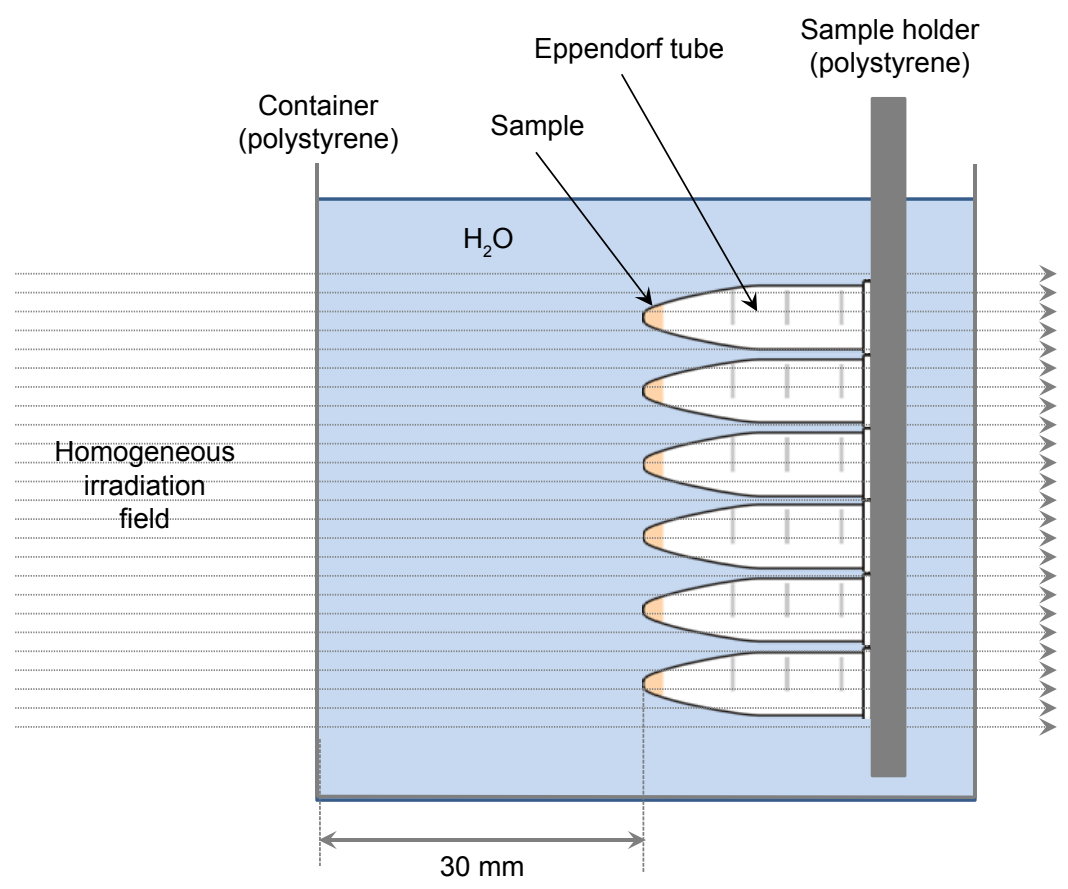

Figure I Sketch of the sample irradiation geometry. 

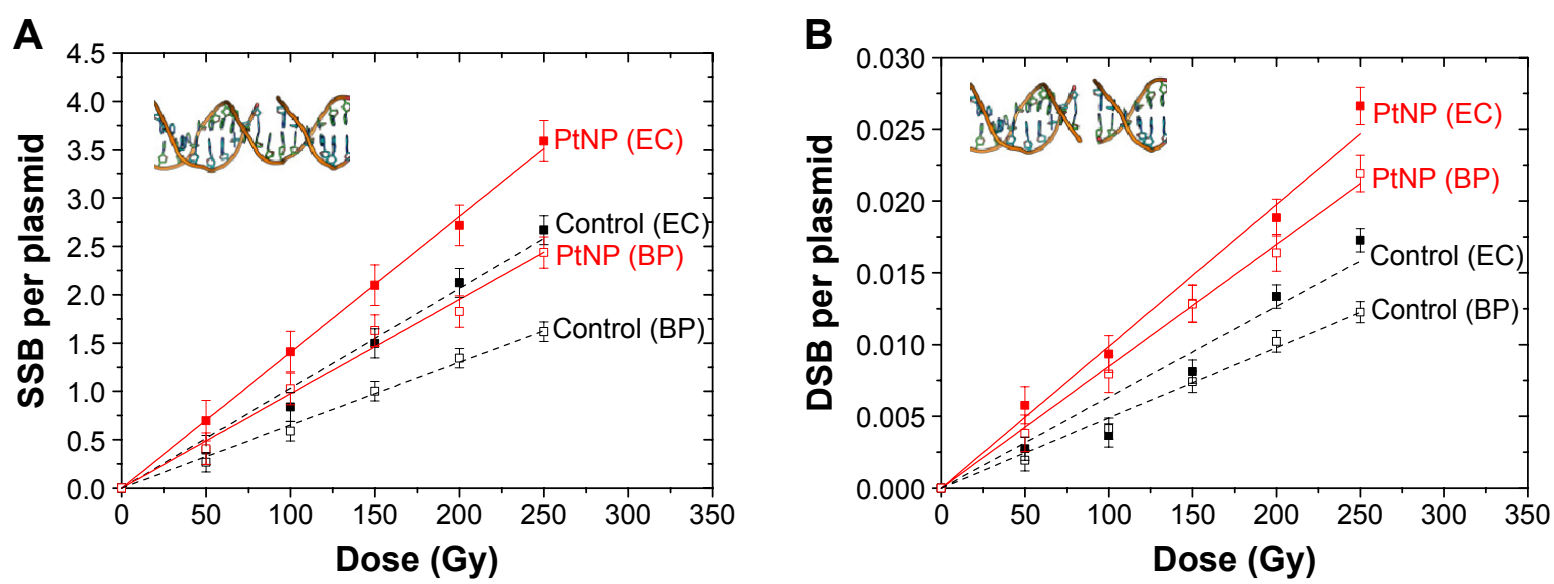

Figure 2 SSBs (A) and DSBs (B) induced in DNA plasmids in the presence of PtNPs (red) and in the control (black) irradiated by protons at the EC and at the BP. Abbreviations: BP, Bragg peak; DSB, double-strand break; EC, entrance channel; PtNP, platinum nanoparticle; SSB, single-strand break.

The yields of SSBs and DSBs were determined as described elsewhere. ${ }^{10,29}$ Briefly, considering that ethidium bromide binds 1.47 times less efficient to supercoiled plasmids $(S)$ than in relaxed $(R)$ and linear $(L)$ forms, we calculated the norm $N=1.47 \times S+R+L$ and then $\mathrm{SSB}=\operatorname{In} \frac{1-L / N}{1.47 \times S / N}$ and $\mathrm{DSB}=\frac{L / N}{1-L / N} \cdot$ No significant artifact due to the binding of NPs to plasmids was found.

\section{Results}

\section{Influence of PtNPs}

The results obtained at 0.44 and $3.6 \mathrm{keV} / \mu \mathrm{m}$ are presented in Figure 2. The curves exhibit linear dose dependences, which are indicative of a single hit mechanism. ${ }^{29}$ For both LETs, the addition of PtNPs significantly increased the number of SSBs and DSBs.

The SSB and DSB yields $\left(m_{\mathrm{SSB}}, m_{\mathrm{DSB}}\right)$, defined as the number of breaks per Dalton and per Gray, are determined using the slopes of the curves. ${ }^{10,29}$ The results are reported in Table 1. We observed that $m_{\mathrm{SSB}}$ and $m_{\mathrm{DSB}}$ increased in the presence of PtNPs. This result demonstrates the properties of PtNPs to amplify the proton radiation effects. This is similar to our previous observation made after carbon ions treatment. ${ }^{10}$
The efficiency of PtNPs was quantified by calculating the amplification factors $\mathrm{AF}_{\mathrm{SSB}}$ and $\mathrm{AF}_{\mathrm{DSB}}$ (Table 1):

$$
\mathrm{AF}_{\mathrm{SSB} / \mathrm{DSB}}=\frac{m_{\mathrm{SSB} / \mathrm{DSB}}(\mathrm{PtNP})}{m_{\mathrm{SSB} / \mathrm{DSB}}(\text { control })}
$$

The AF for DSBs $\left(\mathrm{AF}_{\mathrm{DSB}}\right)$ was significantly higher than for SSBs $\left(\mathrm{AF}_{\mathrm{SSB}}\right)$. This shows that PtNPs are particularly efficient in increasing the production of nanosize lesions, which are most difficult to repair in living cells. This result confirms the results obtained with fast carbon ions used as ionizing radiation. ${ }^{10,29}$

Note that the number of tracks leading to a given deposited dose is eight times lower at high LET $(3.6 \mathrm{keV} / \mu \mathrm{m})$ than at low LET $(0.44 \mathrm{keV} / \mu \mathrm{m})$, which diminishes the geometrical cross section (probability for the ion and its track to activate an NP). This explains the decrease of SSB and DSB at high LET.

Finally, $\frac{m_{\mathrm{SSB}}}{m_{\mathrm{DSB}}}$ was used to characterize the damage quality related to the radiation exposure. For instance, a decrease of this ratio implies a relative increase in DSB production

Table I SSB and DSB yields $\left(m_{\mathrm{SSB}}, m_{\mathrm{DSB}}\right)$ induced by protons $(0.44$ and $3.6 \mathrm{keV} / \mu \mathrm{m})$ in the control and in plasmids with PtNPs

\begin{tabular}{|c|c|c|c|c|c|c|}
\hline \multirow[t]{2}{*}{ Beam LET } & \multirow[t]{2}{*}{ Sample } & \multicolumn{2}{|c|}{ Yields (breaks per Da per Gy) } & \multirow{2}{*}{$\mathrm{AF}_{\mathrm{SSB}}$} & \multirow{2}{*}{$\mathrm{AF}_{\mathrm{DSB}}$} & \multirow{2}{*}{$\frac{m_{\mathrm{SSB}}}{m_{\mathrm{DSB}}}$} \\
\hline & & $m_{\mathrm{SSB}} \times 10^{-10}$ & $m_{\text {DSB }} \times 10^{-11}$ & & & \\
\hline \multirow[t]{2}{*}{$0.44 \mathrm{keV} / \mu \mathrm{m}$} & Control & $36( \pm 1)$ & $2.2( \pm 0.1)$ & - & - & $163( \pm 13)$ \\
\hline & Plasmids + PtNP & $49.8( \pm 0.7)$ & $3.5( \pm 0.1)$ & $1.38( \pm 0.07)$ & $1.6( \pm 0.1)$ & $142( \pm 6)$ \\
\hline \multirow[t]{2}{*}{$3.6 \mathrm{keV} / \mu \mathrm{m}$} & Control & $24.4( \pm 0.7)$ & $1.62( \pm 0.07)$ & - & - & $152( \pm 15)$ \\
\hline & Plasmids + PtNP & $32.5( \pm 0.7)$ & $3.1( \pm 0.1)$ & $1.33( \pm 0.07)$ & $1.9( \pm 0.1)$ & $104( \pm 6)$ \\
\hline
\end{tabular}

Note: The $\frac{m_{\text {SSB }}}{m_{\text {DSB }}}$ ratio and the amplification factors are reported.

Abbreviations: AF, amplification factor; DSB, double-strand break; PtNP, platinum nanoparticle; SSB, single-strand break. 

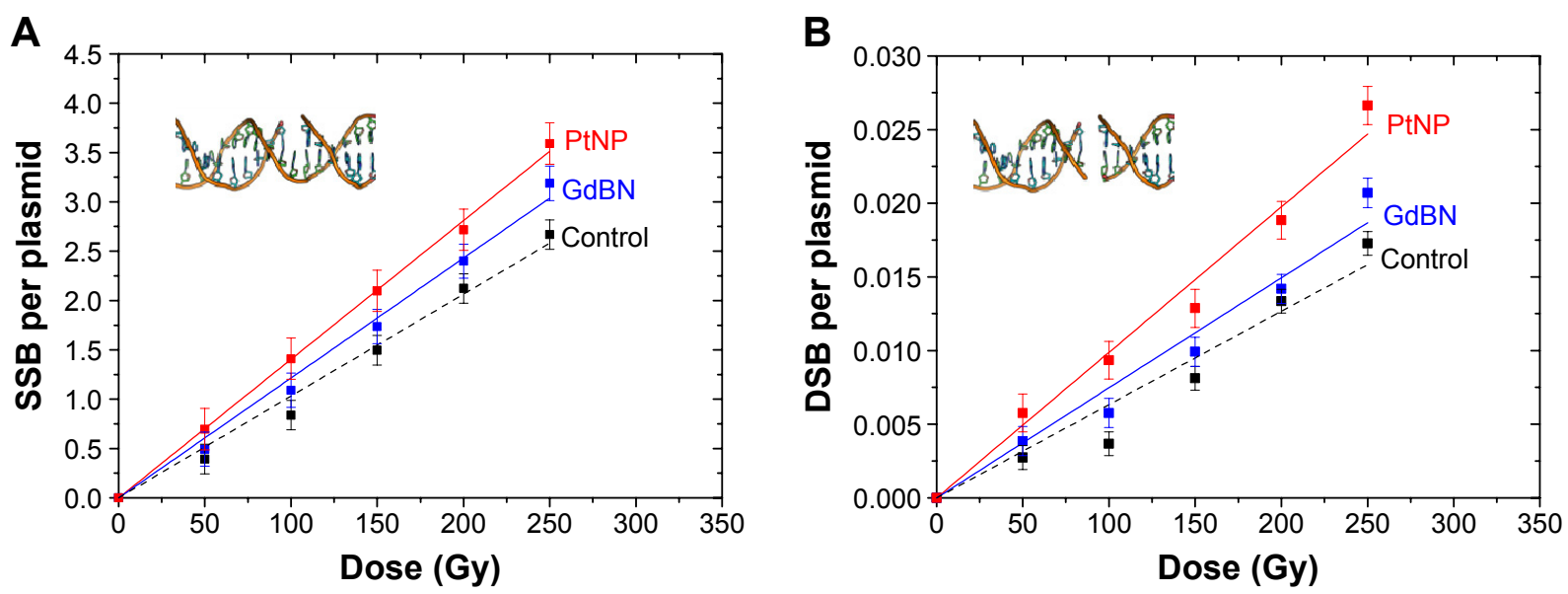

Figure 3 SSBs (A) and DSBs (B) induced by protons at the entrance channel $(0.44 \mathrm{keV} / \mu \mathrm{m})$ in plasmids in the presence of PtNPs $(\square)$ or GdBNs $(\mathbf{\square})$ and in the control $(\mathbf{\square})$ Abbreviations: DSB, double-strand break; GdBN, gadolinium-based nanoparticle; PtNP, platinum-based nanoparticle; SSB, single-strand break.

and indicates an increase in lethality, and we observed such a decrease with increase in the ion beam LET. This confirms the increase of complex damage at the end of the track when the dose increases. Interestingly, the decrease in $\frac{m_{\mathrm{SSB}}}{m_{\mathrm{DSB}}}$ is more pronounced in the presence of PtNPs. Accordingly, the presence of PtNPs not only increases the number of lesions, but also their size and lethality. This effect is even more pronounced at the end of the ion track, which demonstrates the unique properties of PtNPs to improve proton radiation performance by increasing lethality and volume targeting.

\section{Effect of GdBNs}

The effect of GdBNPs was studied under similar conditions as used for PtNPs. The comparison of GdBNs and PtNPs is illustrated in Figure 3 for proton irradiation at $0.44 \mathrm{keV} / \mu \mathrm{m}$ only. The SSB and DSB yields $\left(m_{\mathrm{SSB}}, m_{\mathrm{DSB}}\right), \frac{m_{\mathrm{SSB}}}{m_{\mathrm{DSB}}}$ ratio, and AFs obtained at the two LETs $(0.44$ and $3.6 \mathrm{keV} / \mu \mathrm{m})$ are summarized in Table 2.

As shown in Figure 3, addition of GdBNs amplifies the induction of molecular damages, as observed with PtNPs. However, for the same metal concentration, GdBNs are less efficient than PtNPs in producing complex lesions $\left(\mathrm{AF}_{\mathrm{DSB}}(\mathrm{GdBN})=1.19<\mathrm{AF}_{\mathrm{DSB}}(\mathrm{PtNP})=1.6\right.$ at $0.44 \mathrm{keV} / \mu \mathrm{m}$; $\mathrm{AF}_{\mathrm{DSB}}(\mathrm{GdBN})=1.32<\mathrm{AF}_{\mathrm{DSB}}(\mathrm{PtNP})=1.9$ at $\left.3.6 \mathrm{keV} / \mu \mathrm{m}\right)$.

As observed with PtNPs, the induction of complex damages is more pronounced at the end of the track $(\mathrm{AF}=1.19$ at $0.44 \mathrm{keV} / \mu \mathrm{m}$ and 1.32 at $3.6 \mathrm{keV} / \mu \mathrm{m})$.

\section{Role of water radicals}

In previous studies, we highlighted the major role of ROS in the effect of NPs to amplify radiation-induced damages. ${ }^{10,18}$ In this study, we performed similar experiments with DMSO, a commonly used radical scavenger (see "Materials and methods" section). In Figure 4 is presented the induction of DSBs induced in the presence of PtNPs with and without DMSO at the two LETs under study. A summary of the SSB and DSB yields and related data obtained with PtNPs and GdBNs is presented in Table 3.

For both LETs, the number of molecular breaks (SSBs and DSBs) in samples containing PtNPs and GdBNs decreased dramatically in the presence of DMSO (graphs not shown here). This confirms the major role of hydroxyl radicals as precursors.

Table 2 SSB and DSB yields $\left(m_{\mathrm{SSB}}, m_{\mathrm{DSB}}\right)$ induced by protons at 0.44 and $3.6 \mathrm{keV} / \mu \mathrm{m}$ in the control (pure plasmids) and in plasmids in the presence of GdBNs

\begin{tabular}{|c|c|c|c|c|c|c|}
\hline \multirow[t]{2}{*}{ Beam LET } & \multirow[t]{2}{*}{ Sample } & \multicolumn{2}{|c|}{ Yields (breaks per Da per Gy) } & \multirow[t]{2}{*}{$\mathrm{AF}_{\mathrm{SSB}}$} & \multirow[t]{2}{*}{$A F_{D S B}$} & \multirow{2}{*}{$\frac{m_{\mathrm{SSB}}}{m_{\mathrm{DSB}}}$} \\
\hline & & $m_{\mathrm{SSB}} \times 10^{-10}$ & $m_{\text {DSB }} \times 10^{-11}$ & & & \\
\hline \multirow[t]{2}{*}{$0.44 \mathrm{keV} / \mu \mathrm{m}$} & Control & $36( \pm I)$ & $2.2( \pm 0.1)$ & - & - & $163( \pm 13)$ \\
\hline & Plasmids + GdBN & $42.8( \pm 0.7)$ & $2.65( \pm 0.07)$ & $1.18( \pm 0.03)$ & $1.19( \pm 0.06)$ & $162( \pm 6)$ \\
\hline \multirow[t]{2}{*}{$3.6 \mathrm{keV} / \mu \mathrm{m}$} & Control & $24.4( \pm 0.7)$ & $1.62( \pm 0.07)$ & - & - & $152( \pm 15)$ \\
\hline & Plasmids + GdBN & $23.3( \pm 0.7)$ & $2.15( \pm 0.07)$ & $0.97( \pm 0.03)$ & $1.32( \pm 0.04)$ & $109( \pm 7)$ \\
\hline
\end{tabular}

Note: The $\frac{m_{S S B}}{m_{D S B}}$ ratio and AFs are reported.

Abbreviations: AF, amplification factor; DSB, double-strand break; GdBN, gadolinium-based nanoparticle; SSB, single-strand break. 

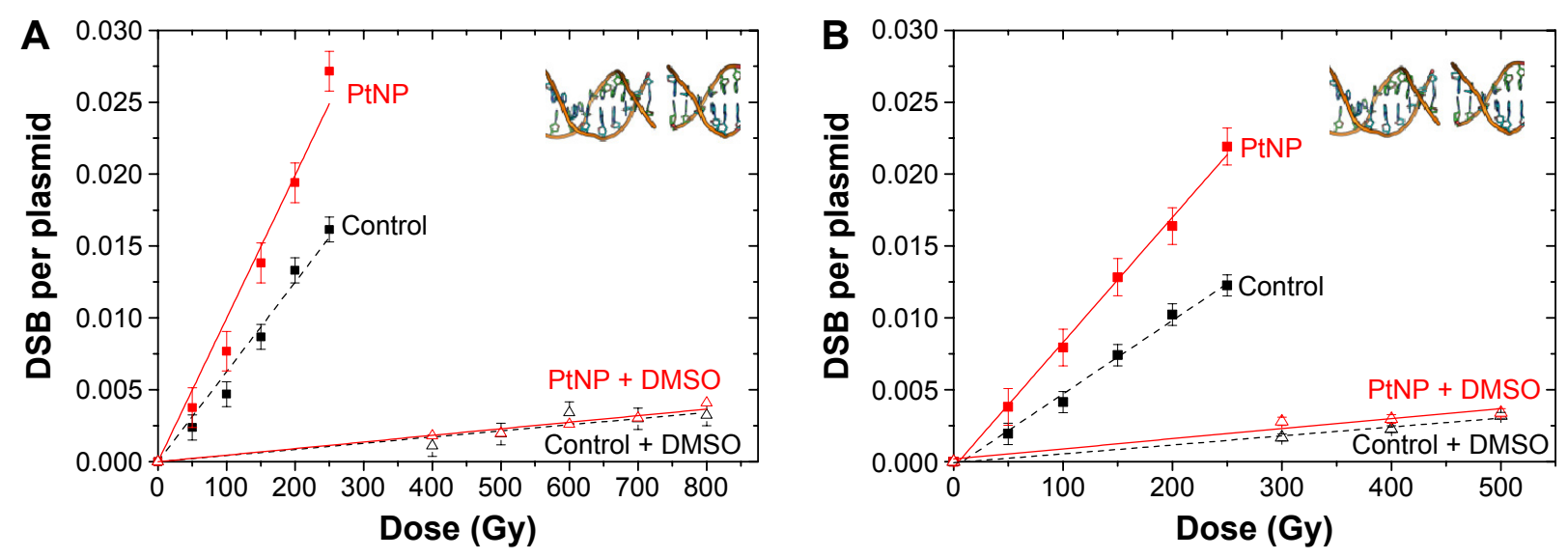

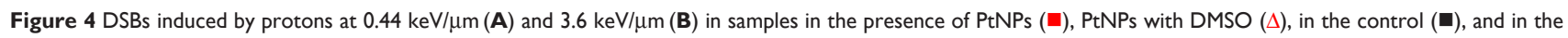
control with DMSO $(\triangle)$.

Abbreviations: DMSO, dimethyl sulfoxide; DSB, double-strand break; PtNP, platinum nanoparticle; SSB, single-strand break.

The contribution of ROS (HO• radicals and by-products) can be quantified as the following ratio (given here for the case of DSBs in the presence of PtNPs):

$$
\mathrm{ROS}^{\bullet}=\frac{m_{\mathrm{DSB}}(\mathrm{PtNP})-m_{\mathrm{DSB}}(\mathrm{PtNP}+\mathrm{DMSO})}{m_{\mathrm{DSB}}(\mathrm{PtNP})}
$$

The damage yields $m_{\mathrm{DSB}}\left(m_{\mathrm{DSB}}\right)$ for PtNPs and GdBNs were extracted from Tables 1 and 2, respectively. The calculated values are reported in the right columns of Table 3. The contributions of hydroxyl radicals are higher than $90 \%$ for PtNPs and GdBNs, which shows that the production of $\mathrm{HO}^{\bullet}$ radicals is a key step in the amplification of radiation effects by the NPs. This finding is similar to our previous results with carbon ions and photons used as ionizing radiations. ${ }^{18,30}$ It is also in agreement with a recent study which evidenced the presence of $\mathrm{HO}^{*}$ radicals in the case of AuNPs activated by high-energy photons..$^{31,32}$ In this context, it is important to note that the dose rate used in our work ( $30 \mathrm{~Gy} / \mathrm{min}$ ) is higher than the dose rates commonly used in therapy (typically $3-7 \mathrm{~Gy} / \mathrm{min}$ ). However, even at $30 \mathrm{~Gy} / \mathrm{min}$, effects such as hydroxyl radical recombination are still low and oxidative stress processes most likely remain unaffected (as opposed to, eg, flash irradiation).

\section{Discussion}

The early-stage processes involved in the amplification of proton radiation effects by metallic NPs have been detailed elsewhere. ${ }^{10,18}$ Briefly, incident ions and secondary electrons produced along the particle track activate NPs by means of Coulombic interaction. The excitation and ionization probability increases with the atomic number of the target. It is higher for platinum $\left(\mathrm{Z}_{\mathrm{Pt}}=78\right)$ than for gadolinium $\left(\mathrm{Z}_{\mathrm{Gd}}=64\right)$.

As shown recently, the excitation of collective electronic modes such as plasmons may also contribute to the activation of metal core NPs (ie, PtNPs) by $1 \mathrm{MeV}$ protons or below. ${ }^{13}$ This effect is expected to be strongly dependent on proton velocity. At the end of the track (in the Bragg peak), where proton velocities are of the order of the velocities of molecular valence electrons, plasmon excitation in metals

Table 3 SSB and DSB yields ( $m_{\text {SSB }}, m_{\text {DSB }}$ ) induced by protons at 0.44 and $3.6 \mathrm{keV} / \mu \mathrm{m}$ in the control (pure plasmids) and in plasmids with PtNPs or GdBNs in the presence of DMSO

\begin{tabular}{|c|c|c|c|c|c|}
\hline \multirow[t]{2}{*}{ Beam LET } & \multirow[t]{2}{*}{ Sample } & \multicolumn{2}{|c|}{ Yields (breaks per Da per Gy) } & \multicolumn{2}{|c|}{ ROS contribution (\%) } \\
\hline & & $m_{\mathrm{sSB}} \times 10^{-10}$ & $m_{\text {DSB }} \times 10^{-11}$ & SSB & DSB \\
\hline \multirow[t]{3}{*}{$0.44 \mathrm{keV} / \mu \mathrm{m}$} & Control + DMSO & $0.97( \pm 0.03)$ & $0.15( \pm 0.01)$ & 97 & 93 \\
\hline & Plasmids + PtNP + DMSO & $1.72( \pm 0.03)$ & $0.16( \pm 0.01)$ & 96 & 95 \\
\hline & Plasmids + GdBN + DMSO & $0.88( \pm 0.04)$ & $0.141( \pm 0.005)$ & 98 & 95 \\
\hline \multirow[t]{3}{*}{$3.6 \mathrm{keV} / \mu \mathrm{m}$} & Control + DMSO & $1.12( \pm 0.02)$ & $0.219( \pm 0.003)$ & 95 & 87 \\
\hline & Plasmids + PtNP + DMSO & $3.05( \pm 0.08)$ & $0.25( \pm 0.02)$ & 91 & 92 \\
\hline & Plasmids + GdBN + DMSO & $0.86( \pm 0.02)$ & $0.17( \pm 0.02)$ & 96 & 92 \\
\hline
\end{tabular}

Note: The contributions of hydroxyl radicals for SSBs and DSBs are reported in the right columns.

Abbreviations: DMSO, dimethyl sulfoxide; DSB, double-strand break; GdBN, gadolinium-based nanoparticle; PtNP, platinum nanoparticle; ROS, reactive oxygen species; SSB, single-strand break. 
can be an important energy deposition channel. At the track entrance, where proton energies are much higher, interactions with single electrons of the medium are expected to dominate (Bethe-Bloch regime).

After the activation step, the electronic de-excitation of NP leads to the emission of electron bursts into the surrounding medium. The de-excitation of collective modes is even higher for atoms rich in $5 \mathrm{~d}$ electrons (ie, platinum). ${ }^{14}$

The interaction of these emitted electrons with water molecules in the medium leads to the production of free radicals ( $\mathrm{HO}^{\bullet}$ as precursor and by-products) concentrated in clusters around the NPs. We, thus, attribute the induction of nanosize molecular damages to the interaction of radical clusters with the plasmid DNA. Note that these nanoscale processes depend exclusively on the electronic properties of NPs and may thus impact all biological targets, including nucleic acids, proteins, and lipids.

As shown before, the amplification of damages by NPs is remarkably stronger at the end of the particle track. For a better representation of this effect, we normalized the damage yield from a per-Gray to a per-track reference. As mentioned before, for a given dose deposition, the number

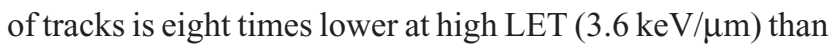
at low LET $(0.44 \mathrm{keV} / \mu \mathrm{m})$. Thus, the relative production of complex breaks at the end and at the entrance of the track is given by $\frac{m_{\mathrm{DSB}}\left(3.6 \mathrm{keV} / \mu \mathrm{m}^{-1} D\right)}{m_{\mathrm{DSB}}\left(0.44 \mathrm{keV} / \mu \mathrm{m}^{-1} D\right)} \times 8$.

For the control, this ratio amounts to $5.8( \pm 0.4)$, which confirms that the production of DSBs increases with the dose deposition along the track (Figure 5). Interestingly, this ratio increases to 6.5 with GdBNs, and even more (7.2) with PtNPs (Figure 5). To our knowledge, this increase of the NP effect with the penetration depth of the incident ion is observed here for the first time. This finding supports the strategy to use NPs to improve not only the quality of the radiation treatment but also the localization of the effect at the end of the track, which is the major goal of proton therapy.

\section{Conclusion}

Using plasmid DNA as molecular probes, for the first time, we presented evidence that the presence of small $(\sim 3 \mathrm{~nm})$ platinum- or GdBNs augments the radiation effects of fast protons. By quantification of molecular damage, we demonstrated that this amplification effect is due to nanoscale processes that induce severe damage in biomolecules. These early-stage mechanisms are very likely characteristic for high-Z NPs in general, such as AuNPs activated by protons, and may explain the amplification of proton action observed in vitro and in vivo previously. ${ }^{21,22}$

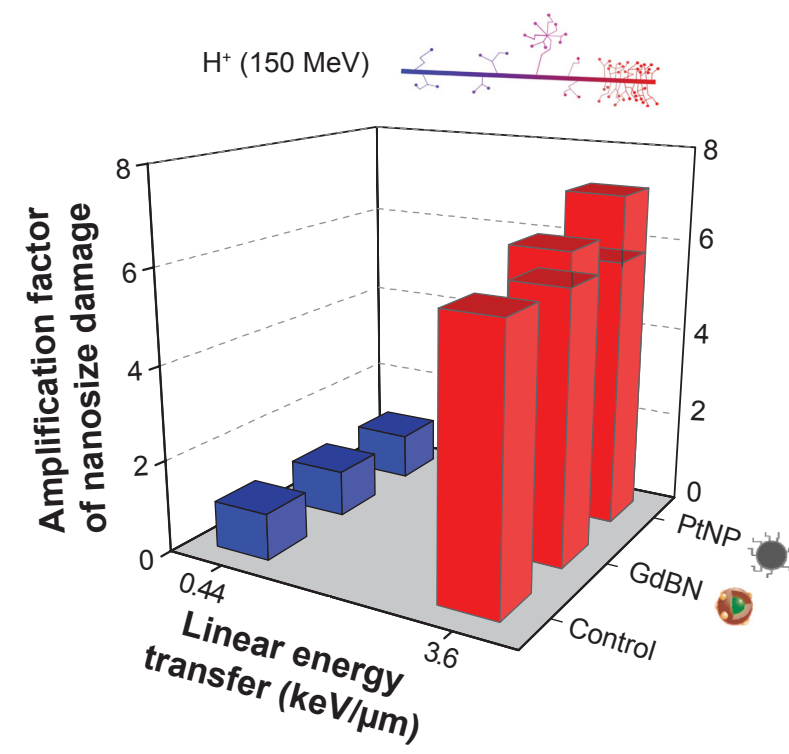

Figure 5 Amplification factor of nanosize molecular damage induced along a $150 \mathrm{MeV}$ proton track in the presence of metal-containing nanoparticles.

Abbreviations: GdBN, gadolinium-based nanoparticle; PtNP, platinum nanoparticle.

The effect is more pronounced in the region of the Bragg peak than in the entrance region of the tracks, which increases the therapeutic potential of these NPs even more. In particular, we established that the presence of NPs enhances the yield of complex nanosize molecular damages ( $>2 \mathrm{~nm}$ ), which is known to be most lethal for cells. The formation of these damages can be attributed to production of radical clusters resulting from NP activation. The fact that stronger radiation damage amplification was found for Pt-based NPs (AF =1.93) than for Gd-based NPs (AF =1.33) can only partly be explained by the differences in atomic number Z. It is very likely that collective electronic excitations (plasmons) play an important role in high-energy proton-NP interactions (as recently predicted theoretically) and are responsible for the superior efficiency of PtNPs. The present study thus confirms the potential of high-Z metalcontaining NPs to increase the efficiency of high-energy proton radiation action, particularly in the tumor volume at the end of the track. Accordingly, combining proton therapy with administration of PtNPs or GdNPs could be a promising strategy for improving proton therapy. Furthermore, the combination of platinum and gadolinium could open theranostic perspectives in proton therapy.

\section{Acknowledgments}

Financial support from the European Union within the COST Action MP1002 "Radiation damage in biomolecular systems: nanoscale insights into ion-beam cancer therapy" (Nano-IBCT) and for the funding of the Marie Curie 
fellowship Nanohapy FP7-PEOPLE-2013-IEF N624370 is gratefully acknowledged.

\section{Disclosure}

The authors report no conflicts of interest in this work.

\section{References}

1. Davis ME, Chen Z, Shin DM. Nanoparticle therapeutics: an emerging treatment modality for cancer. Nat Rev Drug Discov. 2008;7: 771-782.

2. Petros RA, DeSimone JM. Strategies in the design of nanoparticles for therapeutic applications. Nat Rev Drug Discov. 2010;9(8):615-627.

3. Jain PK, El-Sayed IH, El-Sayed MA. Au nanoparticles target cancer. Nano Today. 2007;2(1):18-29.

4. Sun T, Zhang YS, Pang B, Hyun DC, Yang M, Xia Y. Engineered nanoparticles for drug delivery in cancer therapy. Angew Chem Int Ed Engl. 2014;53(46):12320-12364.

5. Hainfeld JF, Slatkin DN, Smilowitz HM. The use of gold nanoparticles to enhance radiotherapy in mice. Phys Med Biol. 2004;49:N309-N315.

6. Kobayashi K, Frohlich H, Usami N, Takakura K, Le Sech C. Enhancement of X-ray-induced breaks in DNA bound to molecules containing platinum: A possible application to hadrontherapy. Radiat Res. 2002; 157(1):32-37.

7. Kobayashi K, Usami N, Porcel E, Lacombe S, Le Sech C. Enhancement of radiation effect by heavy elements. Mutat Res. 2010;704(1-3): $123-131$.

8. Usami N, Furusawa Y, Kobayashi K, et al. Mammalian cells loaded with platinum-containing molecules are sensitized to fast atomic ions. Int J Radiat Biol. 2008;84:603-611.

9. Butterworth KT, Coulter JA, Jain S, et al. Evaluation of cytotoxicity and radiation enhancement using $1.9 \mathrm{~nm}$ gold particles: potential application for cancer therapy. Nanotechnology. 2010;21(29):295101.

10. Porcel E, Liehn S, Remita H, et al. Platinum nanoparticles: a promising material for future cancer therapy? Nanotechnology. 2010;21:85103.

11. McMahon SJ, Hyland WB, Muir MF, et al. Biological consequences of nanoscale energy deposition near irradiated heavy atom nanoparticles. Sci Rep. 2011;1:18.

12. Waelzlein C, Scifoni E, Kraemer M, Durante M. Simulations of dose enhancement for heavy atom nanoparticles irradiated by protons. Phys Med Biol. 2014;59(6):1441-1458.

13. Verkhovtsev AV, Korol AV, Solov'yov AV. Electron production by sensitizing gold nanoparticles irradiated by fast ions. J Phys Chem C. 2015;119:11000.

14. Verkhovtsev AV, Korol AV, Solov'yov AV. Revealing the mechanism of low-energy electron yield enhancement from sensitizing nanoparticles. Phys Rev Lett. 2015;114:063401.

15. Le Duc G, Miladi I, Alric C, et al. Toward an image-guided microbeam radiation therapy using gadolinium-based nanoparticles. ACS Nano. 2011;5:9566-9574.

16. Sancey L, Lux F, Kotb S, et al. The use of theranostic gadoliniumbased nanoprobes to improve radiotherapy efficacy. Br J Radiol. 2014; 87(1041):20140134.
17. Štefančíková L, Porcel E, Eustache P, et al. Cell localisation of gadolinium-based nanoparticles and related radiosensitising efficacy in glioblastoma cells. Cancer Nanotechnol. 2014;5(1):6.

18. Porcel E, Tillement O, Lux F, et al. Gadolinium-based nanoparticles to improve the hadrontherapy performances. Nanomedicine. 2014;10(8): 1601-1608.

19. Durante M, Loeffler JS. Charged particles in radiation oncology. Nat Rev Clin Oncol. 2010;7(1):37-43.

20. Kim J, Seo S, Kim K, et al. Therapeutic application of metallic nanoparticles combined with particle-induced $\mathrm{x}$-ray emission effect. Nanotechnology. 2010;21(42):425102.

21. Polf JC, Bronk LF, Driessen WHP, Arap W, Pasqualini R, Gillin M. Enhanced relative biological effectiveness of proton radiotherapy in tumor cells with internalized gold nanoparticles. Appl Phys Lett. 2011;98: 193702.

22. Kim J, Seo S, Kim H, et al. Enhanced proton treatment in mouse tumors through proton irradiated nanoradiator effects on metallic nanoparticles. Phys Med Biol. 2012;57(24):8309.

23. Vyšín L, Pachnerova Brabcova K, Stepan V, et al. Proton-induced direct and indirect damage of plasmid DNA. Radiat Environ Biophys. 2015; 4(3):343-352.

24. Butterworth KT, Wyer JA, Brennan-Fournet M, et al. Variation of strand break yield for plasmid DNA irradiated with high-Z metal nanoparticles. Radiat Res. 2008;170(3):381-387.

25. Mowat $\mathrm{P}$, Mignot A, Rima $\mathrm{W}$, et al. In vitro radiosensitizing effects of ultrasmall gadolinium based particles on tumour cells. J Nanosci Nanotechnol. 2011;11:7833.

26. Reuvers A, Greensto CL, Borsa J, Chapman J. Studies on mechanism of chemical radioprotection by dimethyl sulfoxide. Int J Radiat Biol Relat Phys Chem Med. 1973;24(5):533-536.

27. Agostinelli S, Allison J, Amako K, et al. GEANT4-a simulation toolkit. Nucl Instrum Methods Phys Res A. 2003;506(3):250-303.

28. International Atomic Energy Agency. Absorbed Dose Determination in External Beam Radiotherapy. An International Code of Practice for Dosimetry Based on Standards of Absorbed Dose to Water. Vol 398. Vienna: IAEA; 2000:135.

29. Usami N, Kobayashi K, Furusawa Y, Frohlich H, Lacombe S, Le Sech C. Irradiation of DNA loaded with platinum containing molecules by fast atomic ions C6+ and Fe26+. Int J Radiat Biol. 2007;83(9):569-576.

30. Porcel E, Kobayashi K, Usami N, Remita H, Le Sech C, Lacombe S. Photosensitization of plasmid-DNA loaded with platinum nano-particles and irradiated by low energy X-rays. JPCS. 2011;261(1):012004.

31. Sicard-Roselli C, Brun E, Gilles M, et al. A new mechanism for hydroxyl radical production in irradiated nanoparticle solutions. Small. 2014;10(16):3338-3346.

32. Misawa M, Takahashi J. Generation of reactive oxygen species induced by gold nanoparticles under x-ray and UV Irradiations. Nanomedicine. 2011;7(5):604-614.
International Journal of Nanomedicine

\section{Publish your work in this journal}

The International Journal of Nanomedicine is an international, peerreviewed journal focusing on the application of nanotechnology in diagnostics, therapeutics, and drug delivery systems throughout the biomedical field. This journal is indexed on PubMed Central, MedLine, CAS, SciSearch $\AA$, Current Contents $\AA /$ Clinical Medicine,

\section{Dovepress}

Journal Citation Reports/Science Edition, EMBase, Scopus and the Elsevier Bibliographic databases. The manuscript management system is completely online and includes a very quick and fair peer-review system, which is all easy to use. Visit http://www.dovepress.com/ testimonials.php to read real quotes from published authors. 